\title{
Encephalopathy Due to Urea Cycle Disorder Post Gastric Bypass Surgery
}

\author{
Toufic Saber ${ }^{1}$, Rabih Braidy ${ }^{2}$, Saleem Abdel Baki ${ }^{3}$, Said Farhat ${ }^{4}$, Alex Malek ${ }^{5}$, Imad EL Hajj ${ }^{5}$ and \\ Mansour El Khoury ${ }^{5}$
}

${ }^{1}$ General Surgery Department, PGY IV - Saint Georges Hospital University Medical Center, Lebanon

${ }^{2}$ Head of division of anesthesia, Anesthesiologist at Khoury general hospital, Lebanon

${ }^{3}$ General Surgery Department, PGY V - Saint Georges Hospital University Medical Center, Lebanon

${ }^{4}$ Gastroenterology Department, Saint Georges Hospital University Medical Center, Lebanon

${ }^{5}$ General Surgery Department, Saint Georges Hospital University Medical Center, Lebanon

*Corresponding author: Mansour el Khoury, Department of General Surgery, Saint George University Medical Center, Lebanon

To Cite This Article: Toufic S, Rabih B, Saleem Abdel B, Said F, Mansour El K. Encephalopathy Due to Urea Cycle Disorder Post Gastric Bypass Surgery. 2020 - 9(5). AJBSR.MS.ID.001439. DOI: 10.34297/AJBSR.2020.09.001439.

Received: 畊 July 09, 2020; Published: 制 July 30, 2020

\begin{abstract}
Obesity is implicated in multiple comorbidities. Moreover, obesity is on the high rise in most of the developed countries due to change of lifestyle and diet habits. Hyper-ammonia causing encephalopathy is rarely seen post-bariatric surgery. We report a 44 years old female with encephalopathy of non-hepatic origin post gastric bypass surgery. This condition was managed successfully with lactulose and low protein diet.
\end{abstract}

Keywords: Hyper-ammonia; bariatric surgeries; lactulose; urea

\section{Introduction}

Obesity is implicated in multiple health care problems including cardiovascular diseases, diabetes and increased frequency of some cancer types such as colon cancer [1-3]. Obesity is on the high rise in developed societies due to lifestyle modifications and diet shifts from healthy diet to junk food and from active life to sedentary living style [4].

In the late $20^{\text {th }}$ century and due to the exponential increase in the burden of obesity on the health care system, bariatric surgeries gained popularity as a solution for such a problem [5]. Gastric bypass surgery (GBS) is considered the benchmark for any weight loss surgery, as it accounted for almost $50 \%$ of all weight loss surgeries performed in 2018 [5,6]. It is prescribed as a management solution for people with morbid obesity having a BMI $>40 \mathrm{Kg} / \mathrm{m}^{2}$ or $>35 \mathrm{Kg} /$ $\mathrm{m}^{2}$ with any other comorbid disease such as diabetes, hypertension and sleep apnea [6].
As any other surgery, complications can be seen in any bariatric surgeries and mostly in GBS7. The most common complications are nutriments and vitamin mal-absorption as well as some neurological derangement $[7,8]$. The neurological problems can be due to ornithine and carnitine deficiencies. Both enzymes are implicated in the urea cycle responsible for transforming ammonia into urea, which is a soluble product, excreted by the body (Figure 1) $[8,9]$. Encephalopathy can be induced by hyper-ammonia, which can be caused either by enzymatic defect in the urea cycle or excess catabolism of proteins $[10,11]$.

In this article a case is presented regarding a urea cycle disorder, which was developed post GBS for one patient, and was managed successfully by lactulose (Figure 1). 


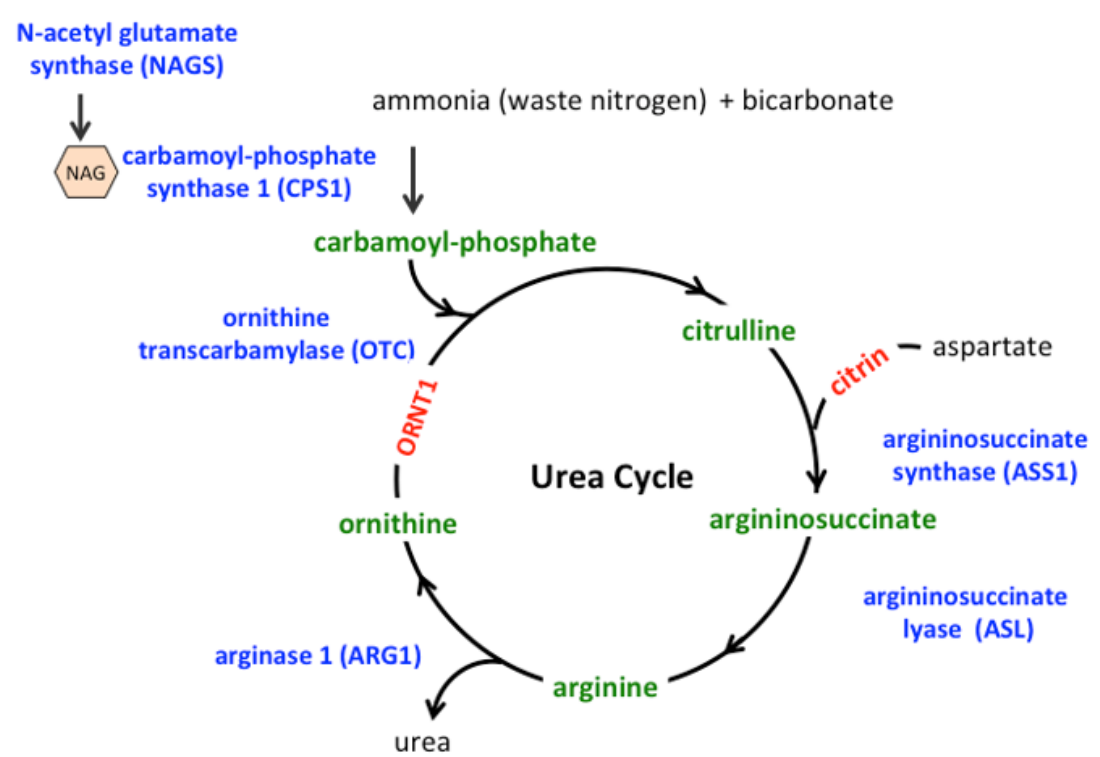

Figure 1: Urea cycle with all the major steps, from Ammonia to Urea. (Reproduced from the website of Rare Diseases Clinical Research Network).

\section{Case presentation}

A 42- year old female with a healthy status was presented to undergo gastric bypass. The Patient's weigh was $110 \mathrm{~kg}$, her height was $157 \mathrm{~cm}$. Her BMI was $44.6 \mathrm{~kg} / \mathrm{m}^{2}$. Her past medical history was positive for arterial hypertension, gastric reflux and migraine. She had gastric sleeve 3 years prior to the present medical event. She is on PPI, propranolol and calcium channel blocker.

Upon admission she had a hemoglobin level of $10.1 \mathrm{mg} /$ dl. The patient underwent Gastric bypass with rouxen Y gastrojejunostomy. No peri or post op complications were encountered. The Upper GI series was done POD4 and it was normal; there was no leak or extra vasation of contrast. On the 4 th day post operation the patient was discharged home on triphasic diet.

Two months post-operation, the patient was presented to the ER with diffuse abdominal pain, nausea and vomiting. She was $95 \mathrm{Kg}$ and her Lab tests were normal except for microcytic anemia (Hb: 10.8 and MCV: 78). A repeat of UGI showed no changes or collections. The patient underwent symptomatic management and she was discharged home on antiemetic and advised to increase fluids intake.

Seven months after, the patient came to the doctor clinic and she was in a cachectic status with a weight of $66 \mathrm{~kg}$ (44 kg weight loss), constant vomiting and abdominal pain post prandial. During this time she had gastroscopy that showed moderate stenosis at the level of the gastro-jejunal anastomosis managed by endoscopic balloon dilatation. The patient was scheduled for surgical revision of the gastro-jejunal anastomosis by open technique. No peri or post op complications were encountered and the UGI was done on POD4 with normal results. The patient was discharged home on POD5, PO intake was fully tolerated.
Three months later the patient was presented to the ER with severe nausea and vomiting. She was unable to perform the 6 minutes walk test. In addition, she was anorexic and not tolerating any PO intake. Laboratory tests were done and showed macrocytic anemia (Hb: 8.2 and MCV: 77) in addition to hypo-albuminimia. She was admitted for parental nutrition and hydration. The patient was diagnosed with anorexia (a psychiatric disorder leading to reduced and rejection of any PO intake). A Physic iatric consult was requested and he put the patient on anti-depressor. The patient was discharged home 7 days later after being able to eat and drink.

At around 1 year from her initial surgery the patient was admitted due to loss of consciousness, mild fever 38.4C and sinus tachycardia of 175 beat per minutes. Her weight was $60 \mathrm{~kg}$. An Intubation was performed in order to protect her airways; and resuscitation was started, in addition to hydration using lactate Ringer, total parental nutrition (1.5L / day), also intravenous digoxin was given few hours later; however, the patient remained unconscious but in sinus rhythm.

The laboratory tests showed that the Levels of vitamin B12 and ferritin in were elevated in addition to a microcytic anemia (Hb: 10.3g/dl and MCV: 74). The patient had normal hemoglucose level on admission. No liver function tests disturbance was signaled on her laboratory tests. The Brain and abdominal CT scan that were done on presentation were within normal limits. The chest X-ray was equivocal for left superior lobe pneumonia, so the patient was started on amoxiclave and levofloxacin. A Neurological assessment and lumbar puncture that were done as well showed normal range with preservation of all the brainstem reflexes.

After 48hours, the patient was still unconscious; however, the ammonia level was highly elevated (180 u/dl) compared to 
the normal range $(15-45 \mathrm{u} / \mathrm{dl})$. The Patient was diagnosed with encephalopathy of non-hepatic origin and was started on lactulose 30cc every 4 hours in the naso-gastric tube. Following the third dose of lactulose the patient regained consciousness and was extubated 24hours later. After extubating the patient, she started PO intake that was tolerated. She was discharged home on lactulose $30 \mathrm{cc}$ twice a day 48 hours later and low protein diet. Follow up was done after 6 months which showed a well nourished female, with a weight of $73 \mathrm{~kg}$, patient is still on lactulose 20cc BID and reports no lethargy, reduced muscles strength, or loss of consciousness.

\section{Discussion}

Obesity is becoming an epidemic in many developed countries due to increase insedentary lifestyle and changes in the nutritional habits [4]. This condition is associated with multiple morbidities and is affecting mostly young people [4]. Due to its effects on social and health of individuals, people tend to consult more and more for bariatric procedures [5]. In general, bariatric procedures is divided into two categories: restrictive (such as insertion of gastric ring or sleeve gastrectomy) and mal-absorptive (such as Gastric bypass and biliary pancreatic shunt) [5,6]. Both types of procedures have side effects and sets of complication that can be faced by the patient and surgeon [7]. Gastric bypass procedure is considered as a bench mark and is used to compare side effects, complications and results for any other bariatric procedures [6].

A case of hyper-ammonia of non-hepatic origin causing encephalopathy post gastric bypass surgery is presented herein. Hyper-ammonia has been previously reported post gastric sleeve surgery but no case has been reported post gastric bypass surgery [11]. Ammonia is a byproduct of catabolism of proteins, it is usually found as Ammonium ion [9]. Most of the ammonium ion is absorbed in the intestine. Ammonium ion is excreted from the human body through the renal system after being convert to urea. Hyper-ammonia can be due either to excess catabolism of proteins or defect in the urea cycle [9]. Hyper-Ammonia if not detected can cause neurological disorders such as encephalopathy and can manifest as vomiting, ataxia, lethargy, tremor, muscle fatigue and unconsciousness $[9,10]$.

Lactulose (beta-galactosidofructose) are nonabsorbable disaccharides that have been in common clinical use since the early 1970s [12,13]. They are degraded by intestinal bacteria to lactic acid and other organic acids [13]. Lactulose appears to inhibit intestinal ammonia production by a number of mechanisms [13]. The conversion of lactulose to lactic acid and acetic acid results in the acidification of the gut lumen [13]. This favors conversion of ammonia (NH3) to ammonium (NH4+); owing to the resultant relative impermeability of the membrane, the NH4+ ions are not easily absorbed, thereby remaining trapped in the colonic lumen, and there is a reduction in plasma NH3 $[13,14]$. Gut acidification inhibits ammoniagenic coliform bacteria, leading to increased levels of non-ammoniagenic lactobacilli. Lactulose also works as a cathartic, reducing colonic bacterial load thus indirectly reducing ammonia level in blood [12].

Encephalopathy due to hyper-ammonia of non hepatic origin post gastric bypass surgery can be safely managed using lactulose. The dose of lactulose initially used was 30cc every 6 hours and patient was managed symptomatically in the immediate post discharge phase. Finding a more scientific rather than symptomatic way to manage these cases should be further studied, in addition discussion of when it is safe to stop lactulose should also be carried.

\section{Conclusion}

Encephalopathy due to hyper-ammonia of non-hepatic origin post gastric bypass is not reported in the literature to our knowledge. Such a case is reported to show that it was managed using lactulose that inhibits the absorption of ammonia in the gut thus reducing its level in blood. Further studies are needed in order to study the dose of lactulose required for maintenance of an adequate level of ammonia level in blood post complications from bariatric surgeries. Finally, early diagnosis and mostly prevention of such complication is needed in order to avoid and reduce morbidity and mortality on the patient.

\section{Acknowledgment}

We would like to acknowledge the efforts of everyone who contributed to the accomplishment of this work, mainly the general surgery department at the saint George hospital Beirut and Khoury hospital Zahle.

\section{Reference}

1. Kachur S, Lavie CJ, De Schutter A, Milani RV, Ventura HO (2017) Obesity and cardiovascular diseases. Minerva Med 108(3): 212-228.

2. Gallagher EJ, Le Roith D (2015) Obesity and Diabetes: The Increased Risk of Cancer and Cancer-Related Mortality. Physiol Rev 95(3): 727-748.

3. Kuipers EJ, Grady WM, Lieberman D, Seufferlein T, Sung JJ, Boelens PG, et al. (2015) Colorectal cancer. Nat Rev Dis Primers 1: 15065.

4. Apovian CM (2016) Obesity: definition, comorbidities, causes, and burden. Am JManag Care 22(7 Suppl): s176-185.

5. Roux CW, Heneghan HM (2018) Bariatric Surgery for Obesity. Med Clin North Am 102(1): 165-182.

6. Wolfe BM, Kvach E, Eckel RH (2016) Treatment of Obesity: Weight Loss and Bariatric Surgery. Circ Res 118(11): 1844-1855.

7. Mesureur L, Arvanitakis M (2017) Metabolic and nutritional complications of bariatric surgery: a review. Acta Gastroenterol Belg 80(4): 515-525.

8. Goodman JC (2015) Neurological Complications of Bariatric Surgery. Curr Neurol Neurosci Rep 15(12): 79.

9. Matsumoto S, Häberle J, Kido J, Mitsubuchi H, Endo F, et al. (2019) Urea cycle disorders-update. J Hum Genet 64(9): 833-847.

10. Zafar A, Khatri IA (2018) An overview of complications affecting the Central Nervous System following bariatric surgery. Neurosciences (Riyadh) 23(1): 4-12. 
11. Kromas ML, Mousa OY, John S (2015) Hyperammonemia-induced encephalopathy: A rare devastating complication of bariatric surgery. World J Hepatol 7(7):1007-1011.

12. Zucker DM, Redulla R (2019) Lactulose Management of Minimal Hepatic Encephalopathy: A Systematic Review. Gastroenterol Nurs 42(1): 84-94.

13. Gluud LL, Vilstrup H, Morgan MY (2016) Non-absorbable disaccharides versus placebo/no intervention and lactulose versus lactitol for the prevention and treatment of hepatic encephalopathy in people with cirrhosis. Cochrane Database Syst Rev 4: CD003044.

14. Zacharias HD, Zacharias AP, Gluud LL, Morgan MY (2019) Pharmacotherapies that specifically target ammonia for the prevention and treatment of hepatic encephalopathy in adults with cirrhosis. Cochrane Database Syst Rev 6(6): CD012334 